\title{
Anti-PD-1 antibodies: effective in GCTs?
}

A small case series recently published in Annals of Oncology investigates immune checkpoint inhibition using anti-PD-1 antibody treatment in patients with platinum-refractory germ cell tumours (GCTs).

Outcomes are poor for patients with platinum-refractory GCTs who relapse after stem cell transplantation or are not suitable for such therapy. Anti-PD-1 antibodies have shown efficacy in some cancers and PD-L1 expression is common in testicular GCTs.

Zschäbitz et al. report on use of anti-PD-1 antibodies in four patients with GCTs who were refractory to platinum and many other treatments. Two patients with PD-L1-negative primary mediastinal yolk sac tumours showed immediate progression following treatment with anti-PD1 antibodies and treatment was ceased. A third patient, with a PD-L1-negative immature teratoma and yolk sac tumour of the left testicle (and liver and bone metastases) received two 3-weekly cycles of anti-PD-1 inhibition; progressive disease was shown at the first staging. Following three further cycles of anti-PD-1 inhibition, staging showed a mixed response and tumour marker decline, but after 10 cycles, staging showed clear progression and anti-PD1 therapy was stopped. A fourth patient with a PD-L1-positive germinoma of the hypophysis received 3-weekly cycles of anti-PD-1 therapy plus the topoisomerase inhibitor etoposide and showed a good partial response after four cycles. After 3 months, staging showed stable disease and etoposide was discontinued while anti-PD-1 therapy was continued. After 15 cycles, staging showed a near complete remission.

The authors says that although immune checkpoint inhibition with anti-PD-1 treatment cannot be recommended for treatment of patients with platinum-refractory GCTs outside of clinical trials, prospective trials are warranted to further evaluate immune checkpoint blockade in such patients.

Rebecca Kelsey

ORIGINAL ARTICLE Zschäbitz, S. et al. Activity of immune checkpoint inhibition in platinum refractory germ cell tumors. Ann. Oncol. http://dx.doi.org/10.1093/annonc/mdw146 (2016) 